Check for updates

Cite this: RSC Adv., 2018, 8, 22729

\title{
Carbon fibre reinforced cellulose-based polymers: intensifying interfacial adhesion between the fibre and the matrix $\uparrow$
}

\author{
László Szabó, (D) *a Sari Imanishi, ${ }^{a}$ Naohiro Kawashima, ${ }^{a}$ Rina Hoshino, ${ }^{a}$ \\ Kenji Takada, $\dot{+}^{\mathrm{a}}$ Daisuke Hirose, ${ }^{a}$ Takayuki Tsukegi, ${ }^{\mathrm{b}}$ Kazuaki Ninomiya ${ }^{\mathrm{c}}$ \\ and Kenji Takahashi (D) *a
}

Interfacial interactions governing the interfacial adhesion between cellulose propionate and carbon fibre surface are placed under scrutiny to pave the way towards the development of green cellulose-based carbon fibre reinforced polymers. A range of molecular entities are deposited on the surface by initially grafting aromatic structures with appropriate functions via diazonium species followed by further derivatization of these entities. Cellulose propionate was also bound covalently to the surface via a tosylated derivative invoking its facile nucleophilic displacement reaction with surface-grafted amino functions. Significant increase in interfacial shear strength was obtained for the cellulose propionategrafted carbon fibre composite as well as for the 4-(aminomethyl)benzene-functionalized sample, in the latter case possible hydrogen bonding took place with the cellulose propionate matrix. Furthermore, the positive effect of a highly lipophilic and yet compact $-\mathrm{CF}_{3}$ substituent was also noted. In order to let the grafted structure efficiently penetrate into the matrix, steric factors, lipophilicity and potential secondary interactions should be considered. It needs to be pointed out that we provide the first synthetic strategy to covalently bind cellulose derivatives to a largely graphitic surface and as such, it has relevance to carbonaceous materials being applied in cellulose-based innovative materials in the future.

Received 21st May 2018

Accepted 14th June 2018

DOI: $10.1039 / \mathrm{c} 8 \mathrm{ra04299c}$

rsc.li/rsc-advances

\section{Introduction}

Carbon fibre reinforced polymers (CFRPs) have emerged as cornerstones for the clean energy supply chain as "lightweighting" plays a key role in improving fuel economy. ${ }^{1}$ Driven by regulatory and consumer-interest pressure, CFRPs have gained appreciable interest in transportation applications relying on high-performance materials with exceptional strength-to-weight ratios.

It has long been recognized that interactions at the fibrematrix interphase govern the stress transfer between the fibre

\footnotetext{
${ }^{a}$ Institute of Science and Engineering, Kanazawa University, Kakuma-machi, Kanazawa 920-1192, Japan. E-mail: szabo-laszlo@se.kanazawa-u.ac.jp; ktkenji@ staff.kanazawa-u.ac.jp; Tel: $+81-76-234-4828$

${ }^{b}$ Innovative Composite Center, Kanazawa Institute of Technology, 2-2 Yatsukaho, Hakusan 924-0838, Japan

'Institute for Frontier Science Initiative, Kanazawa University, Kakuma-machi, Kanazawa 920-1192, Japan

$\dagger$ Electronic supplementary information (ESI) available: Synthetic procedures, characterization of cellulose derivatives including ${ }^{1} \mathrm{H},{ }^{13} \mathrm{C}-\mathrm{NMR}$ and FTIR spectra, Raman and XPS spectra of carbon fibre samples, SEM images of carbon fibres, results of chemical mapping, optical microscopy images, fracture surfaces and cyclic voltammetry data are provided. See DOI: 10.1039/c8ra04299c \$ Present address: Japan Advanced Institute of Science and Technology, School of Materials Science, 1-1 Asahidai, Nomi 923-1211, Japan.
}

and the matrix eventually determining the mechanical performance of the composite. ${ }^{2-6}$ Therefore, a great deal of work has been devoted to what we call now "interphase engineering". ${ }^{7}$ Owing to many pioneering efforts aimed at grafting various structures onto the carbon fibre surface (with no claim of being exhaustive), ${ }^{6-20}$ design rules have been brought to light about tailoring the interphase of thermosetting epoxy composites, as they represent the most widespread application among CFRPs. $^{21,22}$ It is now widely accepted that mechanical interlocking (molecular length and rigidity), covalent interaction with the matrix as well as polarity of the grafted molecule are important factors to increase interfacial shear strength. ${ }^{21}$

Although at present the highest interfacial adhesion has been achieved with thermosetting resins mainly due to the intimate connection of the matrix and the fibre via the crosslinking network, ${ }^{20}$ thermoplastic composites might prevail in the future in many fields due to their inherently greener nature residing in a relatively short production time along with high potential for recyclability. ${ }^{23-25}$ Of thermoplastic polymers, cellulosic materials are undoubtedly superior candidates for fulfilling a sustainable development objective due to their renewability and biocompatibility. ${ }^{26}$ In particular, cellulosic esters have already gained special interest in many innovative composite applications. ${ }^{27}$ Nevertheless, no research has been directed towards cellulose-based CFRPs. Our purpose is to 
intensify the interfacial adhesion between a commercially available cellulose ester and the surface of carbon fibre via establishing covalent linkage in order to pave the way towards green CFRPs yet featuring high performance.

When modification of the largely graphitic surface of carbon fibre is taken into account, it needs to be kept in mind that key mechanical parameters of the fibres should not be detrimentally affected during the grafting process. ${ }^{18}$ Therefore, while in case of carbon nanotubes various chemistries are applied, ${ }^{28}$ only a part of them can be successfully introduced to carbon fibre surface chemistry. ${ }^{29}$ Among the various possibilities, reactions involving diazonium species have caught appreciable scientific interest due to their reliability and versatility in respect to the grafted molecular structure that can have various functionalities opening the gates for many other reactions to further proceed..$^{10,11,17-20,30}$ Moreover, diazonium species can also be electrochemically reduced on the carbon fibre surface, ${ }^{19,20}$ which is highly attractive from the point of view of industrial application as the production of carbon fibres already involves an electrochemical step providing the technological background.

In this study, small molecules are grafted onto the carbon fibre surface via diazonium species followed by further modification of the deposited entity. By grafting an aromatic molecule with a free amino group, tosylated cellulose propionate (CP) could also be covalently attached invoking its facile nucleophilic displacement reaction. We provide the first synthetic strategy to covalently attach a cellulose derivative to a largely graphitic surface. The authors anticipate that the strategy can be introduced to many other carbonaceous materials (e.g. carbon nanotubes) to be applied in bio-based innovative materials in the future.

\section{Experimental}

\section{Materials}

Carbon fibre T700SC-12000-50C was supplied by Toray Industries (Tokyo, Japan). According to the specifications of the producer the fibres have a diameter of $\sim 7 \mu \mathrm{m}$, a tensile strength of $\sim 4900 \mathrm{MPa}$ and 1\% sizing agent on the surface. Therefore, prior to the functionalization procedures the fibres were cleaned to remove sizing agent and any impurities present on the surface. Briefly, the fibres were washed with acetone in an ultrasonic bath twice for $20 \mathrm{~min}$ at $40{ }^{\circ} \mathrm{C}$, rinsed with methanol and water and dried in a vacuum oven (ADP 200, Yamato Scientific Co., Ltd, Tokyo, Japan) at $150{ }^{\circ} \mathrm{C}$ for $3 \mathrm{~h}$. Before each experiments, these fibres were additionally subjected to ultrasonic washing in acetone 5 times for $10 \mathrm{~min}$. The removal of the sizing agent was confirmed by TGA measurement, XPS analysis and AFM measurement (ESI Material, Fig. S1 $\dagger$ ). While the TGA curves show that most of the sizing agent has been removed from the surface, the XPS data clearly indicate the different chemical composition of the surface after the cleaning process. Furthermore, the AFM images also indicate an efficient cleaning process.

Cellulose propionate (approximated $M_{\mathrm{w}}$ was determined to be $200000 \mathrm{~g} \mathrm{~mol}^{-1}$ by gel permeation chromatography according to the producer) was purchased from Scientific Polymer Products, Inc. (Ontario, NY, USA). The degree of substitution (DS) was determined to be 2.76 using the benzoylation procedure as described before (ESI Material, Fig. S4 $\dagger$ ). ${ }^{31}$ Quantitative ${ }^{13} \mathrm{C}$-NMR measurement was also performed to find out the regioselectivity of substitution by integrating the carbonyl carbon peaks (ESI Material, Fig. S5 $\dagger$ ). The $\mathrm{OH}$ group at $\mathrm{C}_{6}$ position is totally substituted ( $\mathrm{DS}=1$ ), while at $\mathrm{C}_{2}$ and $\mathrm{C}_{3}$ the DS value is 0.74 and 0.89 , respectively. FTIR spectrum is shown in Fig. S6. $\dagger$

p-Toluenesulfonyl chloride (tosyl chloride), ortho-dichlorobenzene, tert-butyl nitrite (isoamyl nitrite), 1,4-dioxane, 4-aminobenzoic acid, benzyl chloride and 4-(trifluoromethyl)benzyl chloride were obtained from Tokyo Chemical Industry Co., Ltd. (Tokyo, Japan). Anhydrous pyridine, DMSO and 4-[( $N$-Boc)aminomethyl]aniline were from Sigma-Aldrich (St. Louis, MO, USA) while hydrochloric acid and acetonitrile were from Naclai Tesque, Inc. (Kyoto, Japan). All the other chemicals were provided by Kanto Chemical (Tokyo, Japan).

Synthetic procedures. Synthetic procedures are provided in the ESI Material. $\dagger$

Characterization of cellulose derivatives. Cellulose derivatives were characterized by ${ }^{1} \mathrm{H}$ and ${ }^{13} \mathrm{C}$-NMR spectroscopy in DMSO- $d_{6}$ or $\mathrm{CDCl}_{3}$ as a solvent using a JEOL 400 and $600 \mathrm{MHz}$ FT-NMR spectrometer (Tokyo, Japan). Quantitative ${ }^{13} \mathrm{C}-\mathrm{NMR}$ measurement was carried out at room temperature with 30 seconds pulse-repetition time and with a scan number of 2000 . FTIR spectra were recorded on a Thermo Scientific Nicolet iS10 FTIR spectrometer (Waltham, MA, USA) equipped with a GladiATR accessory (Pike Technologies, Madison, WI, USA) applying a scan number of 128 and a resolution of $2 \mathrm{~cm}^{-1}$.

Characterization of carbon fibre samples. Carbon fibre samples were characterized using thermogravimetric analysis (Shimadzu DTG-60AH, Kyoto, Japan), Raman spectroscopy (Thermo Scientific DXR Raman Microscope, Waltham, MA, USA), X-ray photoelectron spectroscopy (Thermo Scientific KAlpha X-ray Photoelectron Spectrometer System, Waltham, MA, USA), Atomic Force Microscopy (SII NanoTechnology Nanocute, Hitachi, Ltd., Tokyo, Japan) and a JSM-7610F Field Emission Scanning Electron Microscope (FE-SEM) equipped with a JEOL EX-230**BU EX-37001 Energy Dispersive X-Ray Analyser (JEOL, Tokyo, Japan). Composite samples were analysed with a Hitachi S4500 scanning electron microscope (Hitachi, Ltd., Tokyo, Japan) and an Olympus BX50 microscope (Tokyo, Japan) equipped with polarizing lens. Details of the experimental setup of these techniques are provided in the ESI Material. $\dagger$

Physical characterization - fragmentation test. Fragmentation test was conducted with single fibre composites prepared from $\mathrm{CP}$ and the corresponding functionalized/unfunctionalized carbon fibre $(\sim 20 \mathrm{~cm}$ long carbon fibre samples were initially prepared for this test) using a hydraulic hot press machine (Type MH-10, Imoto Machinery Co., Kyoto, Japan). The experimental procedure is outlined in Fig. S2 and S3 in the ESI Material. $\dagger$ The prepared specimens were strained longitudinally with 0.5 $\mathrm{mm} \min ^{-1}$ crosshead speed using a Shimadzu Autograph AG-X Plus $5 \mathrm{kN}$ tensile tester (Kyoto, Japan). The fragmentation process was monitored real time with a high-resolution digital camera (N.O.W.-D2X3Z-KSH, Nihonkouki, Aoki, Japan). The 
fragment size was determined after saturation occurred (no fragmentation anymore) using AUSB-K version 14.4 program (Nihonkouki, Aoki, Japan) calibrated with an objective micrometre (Shibuya Optical Co., Ltd., Wako, Japan). The apparent interfacial shear strength $(\tau)$ can be calculated according to the Kelly-Tyson model (1): ${ }^{32}$

$$
\begin{gathered}
\tau=\frac{\sigma_{\mathrm{fu}} d}{2 l_{\mathrm{c}}} \\
l_{\mathrm{c}}=\frac{4}{5} l \\
\sigma_{\mathrm{fu}}=\sigma_{\mathrm{l}}\left(\frac{l_{1}}{l_{\mathrm{c}}}\right)^{\frac{1}{m}}
\end{gathered}
$$

where $\sigma_{\mathrm{fu}}$ is the fibre tensile strength at the critical length, $d$ is the fibre diameter and $l_{\mathrm{c}}$ is the critical fragment size, which can be calculated from the average fragment size $(l)$ according to Ohsawa's relationship (2). ${ }^{33}$ The strength of the fibre at the critical length can be calculated according to eqn (3), where $\sigma_{1}$ is the average single fibre tensile strength at a gauge length of $l_{1}$ and $m$ is the Weibull modulus estimated from the single fibre tensile test data. ${ }^{29,34,35}$ The details of the calculation can be found in the ESI Material (Fig. S3†). A minimum of ten composite samples were prepared from each functionalized/unfunctionalized carbon fibres. Statistical analysis was performed to determine significant differences $(\alpha=0.05)$ between the interfacial shear strength values using the multiple $t$-test function of GraphPad Prism 7 (GraphPad Software, La Jolla, CA, USA).

\section{Results and discussion}

In the beginning of our research, we have basically designed two synthetic pathways to make carbon fibre surface meet cellulose chemistry. Both of these pathways are based on the ground that carbon fibre surface can be functionalized efficiently via diazonium species to introduce reactive functionalities in respect to cellulose and its derivatives. One reaction path involves an amino group on the surface able to take part in a nucleophilic displacement reaction with a tosylated cellulose derivative (Scheme 1A). The second reaction route invokes the high reactivity of the hydroxyl groups of cellulose derivatives towards acyl chlorides and necessitates the initial generation of a carboxylic acid moiety on the carbon fibre surface (Scheme 1B).

We have chosen commercially available cellulose propionate as a promising candidate for the cellulose-based matrix of the composite and as a starting material for the covalent modification of the carbon fibre surface. Our choice was based on the superior characteristics of this commercial product compared to other cellulose esters available on the market (high molecular weight providing processability at high temperature and increased tensile strength).

\section{Grafting small molecules via diazonium species}

When solid phase chemistry is considered, as it is the case for carbon fibres, it needs to be recognized that steric effects have
A

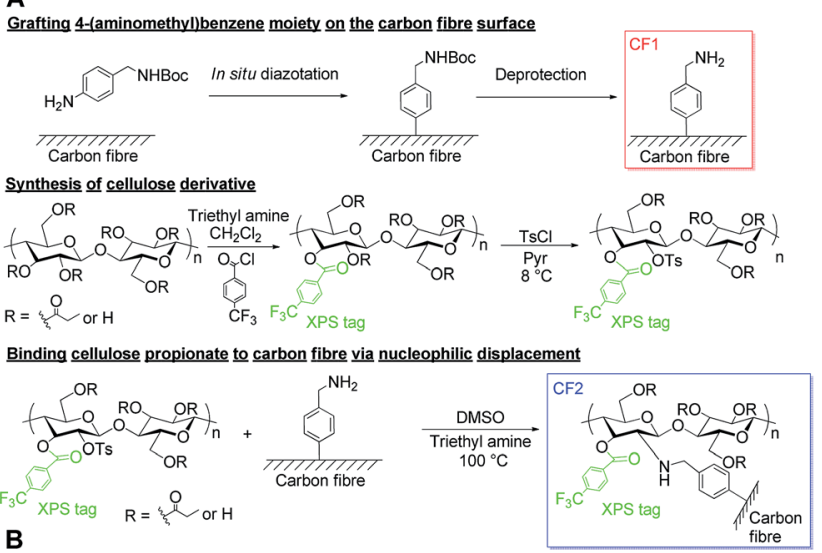

Grafting benzoyl chloride moiety on the carbon fibre surface

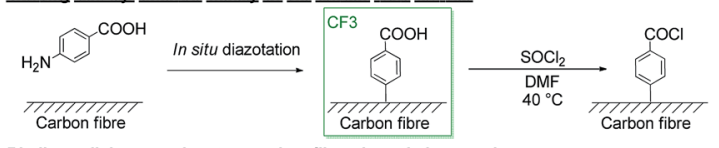

Binding cellulose propionate to carbon fibre via acylation reaction

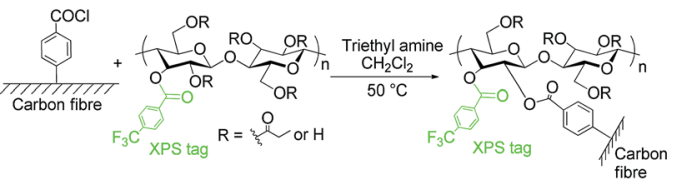

Scheme 1 Synthetic strategies to covalently bind cellulose propionate to the carbon fibre surface. Nucleophilic displacement (A) and acylation approach (B)

an increasing impact compared with homogeneous solutions. ${ }^{18}$ From this point of view, we were especially interested in the degree of substitution (DS) value and the distribution of free hydroxyl groups along the cellulose backbone in commercially available cellulose propionate, chosen to be our candidate for the matrix of the composite. The DS value is determined to be 2.76 for the CP sample (Fig. S4, ESI Material $\dagger$ ), and the free hydroxyl groups are distributed along the anhydroglucose unit as follows: $\mathrm{DS}_{\mathrm{C} 6}=1, \mathrm{DS}_{\mathrm{C} 3}=0.89$ and $\mathrm{DS}_{\mathrm{C} 2}=0.74$ (Fig. S5, ESI Material $\dagger$ ). Saturation at the $\mathrm{C}_{6}$ position means that reaction can only take place close to the cellulose backbone surrounded by propionate units. This issue has raised some concerns among us in respect to steric hindrance especially for the nucleophilic displacement reaction. Therefore, to facilitate this process, we have decided to use 4-(aminomethyl)aniline rather then $p$-phenylenediamine as starting molecule for the functionalization (Scheme 1A). It is also due to steric reasons that we intended not to build on defect site carboxyl groups as it can be found in several studies, ${ }^{\mathbf{8 , 9}, 24,29}$ and rather used 4-aminobenzoic acid as starting compound for reaction path B (Scheme 1B).

The functionalization reaction proceeds via in situ generated diazonium species using the corresponding amine and isoamyl nitrite as the agent providing $\mathrm{NO}^{+}$for the diazotization. The diazonium species are not isolated, however, decomposed by heating to generate highly reactive radical species that can react with the electron-rich graphitic surface of carbon fibre. In order to avoid side reactions, the primary amino group of 4-(aminomethyl)aniline needs to be protected under our conditions. However, this might not be the case below $\mathrm{pH}=9.7$ in water as 
the primary amino group would be anyway protonated $\left(\mathrm{p} K_{\mathrm{a} 1}=\right.$ $\left.3.74, \mathrm{p} K_{\mathrm{a} 2}=9.70\right){ }^{36}$ tert-Butoxycarbonyl (Boc) protecting group was applied since the protection/deprotection step had been elaborated and optimized previously for carbon fibre. ${ }^{18}$

Detection of small molecules grafted on the carbon fibre surface is a quite challenging task as the carbon fibre structure is heterogeneous and includes common organic elements (C, O, $\mathrm{N})$. One of the most powerful techniques to date is XPS detection as it analyses a very narrow layer of the surface (typically 0$10 \mathrm{~nm}$ ) suppressing other elements from the bulk carbon fibre (having a diameter of $\sim 7 \mu \mathrm{m}$ ). After carefully examining highresolution XPS spectra or introducing XPS-tags containing atoms that are not native to the carbon fibre surface, the limitations could be overcome in several cases., ${ }^{8,9,11,17-20,24,29} \mathrm{It}$ is also important to acquire XPS spectra from different locations along the carbon fibre with as large spot size as possible to reduce the effects arising from heterogeneity (in our case a spot size of 400 $\mu \mathrm{m}$ was used).

Fig. 1 shows the high-resolution C1s spectra of control carbon fibre (A) along with the functionalized carbon fibre derivatives CF3 and CF1 (Fig. 1B and C, respectively). Control fibre denotes carbon fibre sample that was subjected to the same treatment as functionalized fibre, however, without reagents added. In the course of our study, we recognized that this type of sample is the most comparable with our results, and it is the best way to represent the reality. The C1s spectrum of the control fibre consists of a rather broad peak that was not further resolved, however, covered overlapping spectrum of localized amorphous carbon as well as extensively delocalized C $\mathrm{sp}^{2}$ structures of the bare carbon fibre surface. ${ }^{8,11,37}$ The broad peak at $289.88 \mathrm{eV}$ can be assigned to defect sites with $-\mathrm{COOH}$ and N-components (imperfect "defect sites" are introduced during the production process of carbon fibre), ${ }^{29}$ and contribution of $\pi-\pi^{*}$ shake-up transitions are also expected. ${ }^{37}$ The high-resolution C1s spectrum of CF3 functionalized carbon fibre (Fig. 1B) is markedly different from the bare carbon fibre (Fig. 1A) with a sharp peak indicating appreciable contributions from delocalized C-C bonds (Fig. 1B). Besides the small peak at $288.38 \mathrm{eV}$ probably arising from defect sites $(-\mathrm{COOH}$ and $\mathrm{N}$ component being introduced during the production process of carbon fibres), ${ }^{29}$ two additional peaks can be observed at $287.18 \mathrm{eV}$ and $289.48 \mathrm{eV}$ representing new $-\mathrm{C}=\mathrm{O}$ and $-\mathrm{COOH}$ functions on the surface. ${ }^{8,11}$ Furthermore, in the C1s spectrum of CF1 functionalized carbon fibre (Fig. 1C), the peak at $286.68 \mathrm{eV}$ provides evidence for the presence of $\mathrm{C}-\mathrm{N}$ bonds on the surface. ${ }^{8}$ The peak at $289.28 \mathrm{eV}$ can again be assigned to defect sites. These results clearly confirm that the desired entities are deposited on the carbon fibre surface.

In addition to XPS characterization, we have also obtained the Raman spectra of these carbon fibre samples. The Raman spectra of carbon black materials are sensitive probes to detect surface defects and disorders by following the characteristic $\mathrm{D}$ band (defects) and $\mathrm{G}$ band (graphitic structure) intensities at $\sim 1350$ and $\sim 1610 \mathrm{~cm}^{-1}$, respectively (for Raman spectra see Fig. S10 in the ESI Material $\dagger$ ). ${ }^{10,11,30,37,38}$ By integrating the peak area of these bands, $I_{\mathrm{D}} / I_{\mathrm{G}}$ was calculated to be 1.9, 2.92 and 2.45 for the unfunctionalized, benzoic acid-grafted (CF3) and 4-
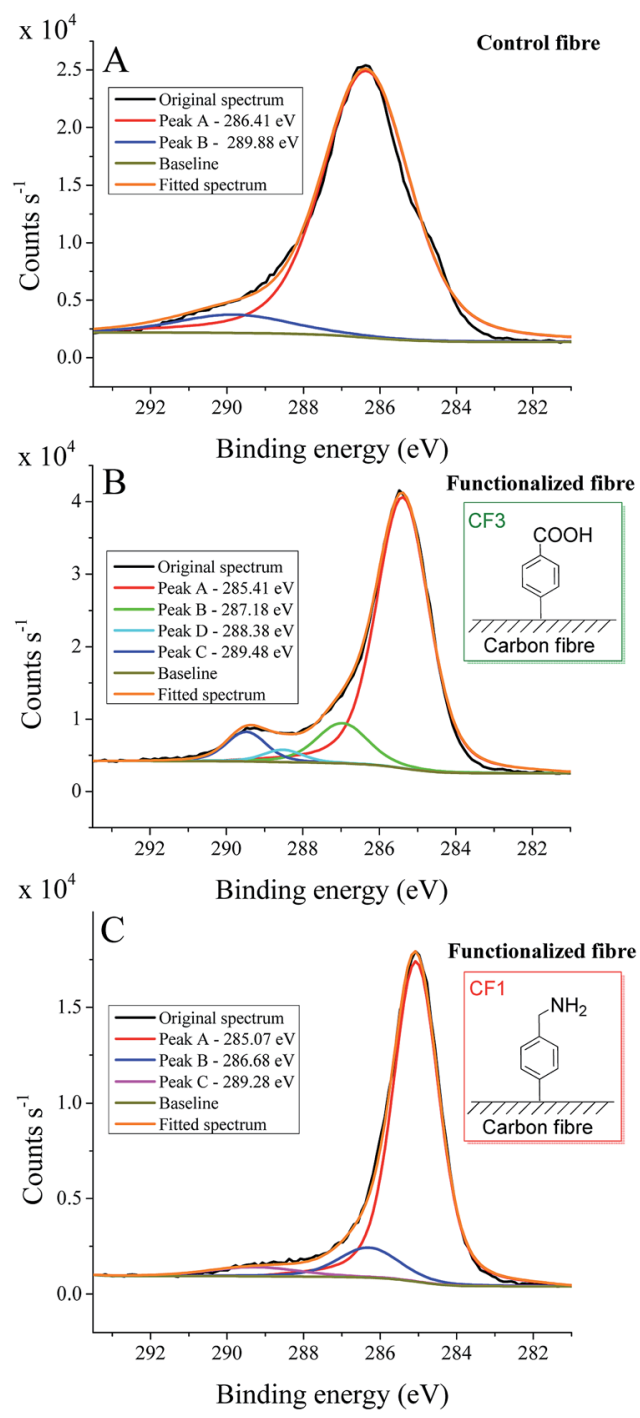

Fig. 1 High-resolution C1s spectra with curve fitting for control sample (A), functionalized carbon fibre CF3 (B) and CF1 (C).

(aminomethyl)benzene-functionalized (CF1) samples, respectively. This finding also clearly supports a successful functionalization process.

\section{Binding cellulose propionate to carbon fibre}

Since cellulose propionate also consists of carbon and oxygen, its detection on the carbonaceous surface is challenging and we are limited only to the high-resolution C1s spectrum, where different oxidation states and chemical environments of a carbon atom can be observed. In the course of our research, we intended to give a unequivocal evidence of a successful grafting procedure and therefore, we decided to incorporate an XPS-tag into the CP structure. We have chosen the trifluoromethyl group for such a purpose and performed acylation reaction with 4-(trifluoromethyl)benzoyl chloride to afford the XPS-tagged cellulose propionate derivative (shown in Scheme 1A) with a $\mathrm{DS}_{\mathrm{XPS}}$-tag of $0.034\left({ }^{1} \mathrm{H}-\mathrm{NMR}\right.$ spectrum is shown in Fig. S7 $\dagger$ ). Only 0.1 equivalent of the reagent per anhydroglucose 
unit was applied to keep the $\mathrm{DS}_{\mathrm{XPS}}$-tag low in order to avoid any interference with the functionalization process. The trifluoromethyl group has been successfully applied as XPS-tag in previous studies. ${ }^{\mathbf{1 7 , 1 8}}$

This XPS-tagged cellulose propionate was then further tosylated in case of the nucleophilic displacement reaction (Scheme 1A) or used directly for the acylation process on the carbon fibre surface (Scheme 1B). The tosylation of CP was carried out with excess amount of tosyl chloride (3 equivalent per anhydroglucose unit) to obtain high $\mathrm{DS}_{\text {tosyl }}$. The reaction proceeded smoothly to afford the tosylated XPS-tagged CP with a $\mathrm{DS}_{\text {tosyl }}$ of $0.147\left({ }^{1} \mathrm{H}\right.$ and ${ }^{13} \mathrm{C}-\mathrm{NMR}$ spectra are shown in Fig. S8 and S9, $\dagger$ respectively). The nucleophilic displacement reaction was then performed with the tosylated XPS-tagged cellulose propionate and the 4-(aminomethyl)benzene-functionalized carbon fibre (CF1) under similar conditions that were used previously for this type of reaction of cellulose derivatives (Fig. 1A). ${ }^{39}$

The acylation reaction (Scheme 1B) was carried out by firstly preparing the acyl chloride from the benzoic acidfunctionalized carbon fibre (CF3) with thionyl chloride and DMF (as catalyst) (experimental procedure is shown in the ESI Material, pp. S7 and $8 \dagger$ ). The functionalized carbon fibre sample thus prepared was washed with dichloromethane and directly used for the acylation step with the XPS-tagged cellulose propionate to avoid decomposition of the reactive acyl chloride prior to the experiment. Unfortunately, despite our best efforts to prepare the desired CP-functionalized carbon fibre, the reaction did not take place based on our XPS analysis. The underlying reason might originate from the instability of the acyl chloride reacting with impurities either absorbed on the carbon fibre surface or present in the cellulose propionate derivative. It should be noted that similar functionalization could be accomplished in other cases in the literature when defect site $-\mathrm{COOH}$ groups were involved in the reaction (in our case this was avoided due to steric considerations). ${ }^{8}$ Therefore, we anticipate that trace amount of water in the cellulose derivative can be responsible for this phenomenon.

Fortunately, the nucleophilic displacement reaction is not sensitive to water and leads to successful functionalization (Scheme 1A). Fig. 2 shows the high-resolution F1s (A) and C1s (B) spectra of the CF2 functionalized carbon fibre sample, the survey spectrum with elemental composition is shown in Fig. S11 (ESI Material $\dagger$ ). Besides clear evidence of the presence of fluorine (Fig. 2A) and thereby the successful covalent attachment of CP to the carbon fibre, the C1s spectrum also gives supporting evidence (Fig. 2B). The main carbon peak centred at $285.04 \mathrm{eV}$ is rather sharp compared to the control carbon fibre sample (Fig. 1A), and again indicates the presence of $\mathrm{C}-\mathrm{C}$ bonds on the surface in different chemical environment (distinct localized and delocalized $\mathrm{C}-\mathrm{C}$ bonds). ${ }^{\mathbf{8 1 1}, 37}$ The band peaking at $285.88 \mathrm{eV}$ can be attributed to $\mathrm{C}-\mathrm{N}$ and $\mathrm{C}-\mathrm{N}-\mathrm{C}$ bonds. ${ }^{8}$ Furthermore, the peak at $287.17 \mathrm{eV}$ arises from $\mathrm{C}=\mathrm{O}$ components of the propionate units of the cellulose derivative. ${ }^{8}$ The band at $289.48 \mathrm{eV}$ partly belongs to defect sites with $-\mathrm{COOH}$ and $\mathrm{N}$-components (with contribution of $\pi-\pi^{*}$ shake-up transitions), ${ }^{37}$ and its apparently broad feature suggests some other $\mathrm{C}-\mathrm{O}$ bonds probably originating from cellulose propionate.
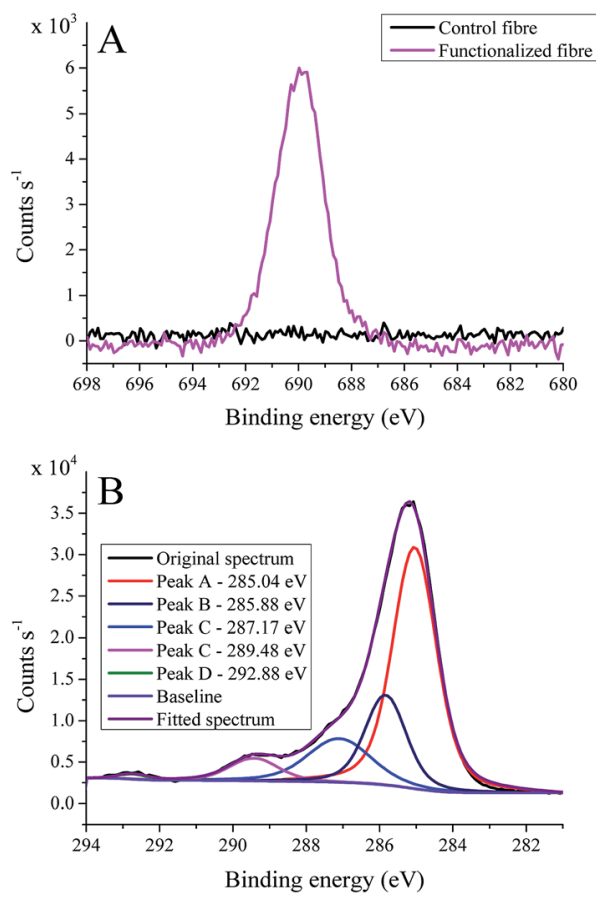

Fig. 2 High-resolution F1s (A) and C1s (B) spectra of cellulose propionate-functionalized carbon fibre samples (CF2).

Furthermore, there is also a small peak observable at $292.88 \mathrm{eV}$ that can be assigned to the $-\mathrm{CF}_{3}$ moieties, however, the contribution of $\pi-\pi^{*}$ shake-up transitions cannot be excluded. ${ }^{40}$

\section{Surface topography of carbon fibres}

It is also of our prime interest to apply chemistries that do not detrimentally affect single fibres as it would be devastating for the final performance of the composite. Therefore, we have designed the synthetic strategy to involve reactions that are proved to be applicable for our purpose, including the grafting procedure of small molecules and the deprotection step. ${ }^{18}$ To further prove that single fibres are physically not damaged we have performed FE-SEM-EDS experiments. In the course of these measurements FE-SEM image is first acquired and then an in-depth chemical mapping is performed (the penetration of the electron beam is few $\mu \mathrm{m}$ and the diameter of the carbon fibre is $7 \mu \mathrm{m})$. It is also due to the high penetration depth that EDS measurement is not appropriate for surface analysis of grafted molecules on carbon fibre surface, however, XPS is the method of choice (0-10 nm analysis depth).

Fig. S12 (ESI Material $\dagger$ ) shows the FE-SEM images of the carbon fibre samples, the EDS chemical mapping can be found in the ESI Material (Fig. S13-16†). There is no physical damage observable on the fibres and according to the chemical mapping they preserve their integrity. The images indicate that there is a homogenous and smooth coating on the surface for the carbon fibre grafted with cellulose propionate (CF2) (Fig. S12B $\dagger$ ), and in case of the benzoic acid (CF3) (Fig. S12C $\dagger$ ) and 4-(aminomethyl)benzene-grafted (CF1) (Fig. S12D †) fibres the surface exhibits roughness to a small extent, as expected. 
Derivatization of benzoic acid (CF3) and 4-(aminomethyl) benzene-functionalized (CF1) carbon fibres

In an attempt to understand the effects that govern the interfacial adhesion between the CP matrix and the carbon fibre surface, we decided to systematically modify the functionalized samples to introduce side chains with different degree of lipophilicity and conformational freedom. In the course of these experiments, it was recognized that confirmation of successful modification is difficult and we need to validate the proceeding of these reactions with model molecules.

For this purpose, to verify the acylation of surface-bound 4(aminomethyl)benzene (CF1) the previously used 4-(trifluoromethyl)benzoyl chloride was applied, while alkylation of surface-bound benzoic acid (CF3) was probed by using 4-(trifluoromethyl)benzyl chloride.

The completion of these reactions could barely be followed by obtaining the high-resolution F1s spectrum due to the low intensities compared to signal noise (Fig. S24, ESI Material $\dagger$ ). Nevertheless, the high-resolution C1s spectra gave satisfying information about successful derivatization. The functionalization is confirmed indirectly as those peaks assigned to $\mathrm{C}-\mathrm{N}$ components and $-\mathrm{COOH}$ groups (see Fig. $1 \mathrm{C}$ and $\mathrm{B}$, respectively) disappear in the spectra of the newly functionalized derivatives (see Fig. S25A and $\mathrm{B}, \uparrow$ respectively).

After this proof-of-concept experiments a range of derivatives were synthesized for interfacial shear strength experiments using benzoyl chloride, propionyl chloride, decanoyl chloride as acylating agents, and benzyl chloride as alkylating agent. The functionalized derivatives were also subjected to FE-SEM-EDS investigations to confirm that fibres were not damaged during the experiments. The results of the FE-SEM-EDS analysis are shown in the ESI Material for all of the functionalized derivatives (Fig. S17-23†).

\section{Interfacial shear strength determination}

Interfacial shear strength (IFSS) was determined using a range of successfully functionalized carbon fibre derivatives and neat cellulose propionate as a matrix. It shall be recognized that interfacial shear strength is not only determined by the interphase, however, the matrix itself also plays a role by efficiently transferring the stress to the interphase. From this point of view, CFRPs based on epoxy resins have the advantage of the crosslinking network while we are somewhat limited by secondary interactions between the interphase and the CP matrix.

Fig. 3 shows the IFSS values for unfunctionalized/ functionalized carbon fibre composites. Neat carbon fibre denotes carbon fibre T700 as received, with sizing agent on the surface (control fibre was defined above). Furthermore, for IFSS experiments cellulose propionate-functionalized carbon fibre was prepared without the XPS-tag. It is apparent from Fig. 3 that there is slight increase in the IFSS value as the sizing agent is removed from the carbon fibre surface probably due to the unfavourable interaction between the polar groups of the sizing agent and the alkyl chains in CP. The negative effect of polar groups on the surface can be further substantiated with the result obtained for the benzoic acid-functionalized (CF3) carbon fibre composite

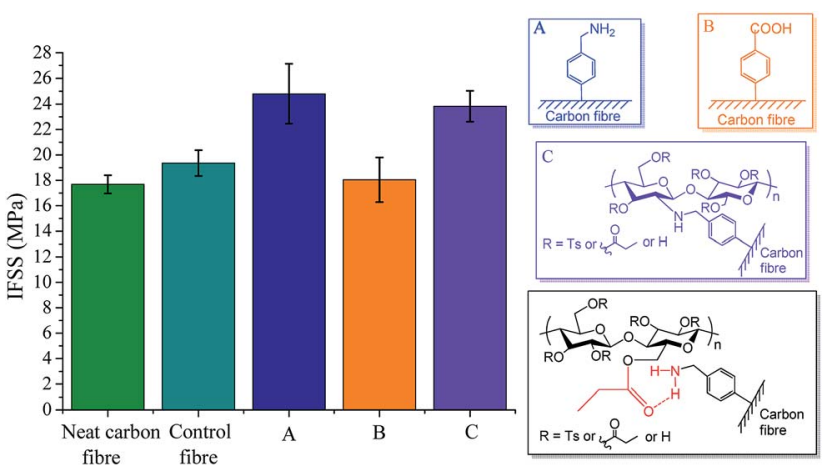

Fig. 3 Interfacial shear strength (IFSS) of carbon fibre reinforced cellulose composites - effect of functionalization.

(Fig. 3B). Significant increase in the IFSS can be observed for the cellulose propionate-functionalized carbon fibre composite, as expected. Surprisingly, similar IFSS value could be obtained for the 4-(aminomethyl)benzene-functionalized (CF1) carbon fibre composite. This phenomenon might be attributed to favourable hydrogen bonding interactions taking place between the amino group of the grafted molecule and the carbonyl oxygen of the propionyl unit of CP, as it is shown in Fig. 3.

The presence of 4-aminophenyl group on the carbon fibre surface has been reported to be beneficial in several cases, e.g. for epoxy and copoly(phthalazinone ether sulfone) composites. $^{\mathbf{1 0 , 1 9 , 3 0}}$ Our finding shows that similar function is also advantageous for a cellulose-based matrix. Although grafting the matrix itself to the carbon fibre surface did not lead to further improvement, the authors would like to highlight the range of possibilities their synthetic approach offers. By using this strategy one is able to immobilize cellulose derivatives on any kind of graphitic surfaces that might give rise to innovative green materials in the future.

It has been revealed in previous studies that molecular length, rigidity and conformational freedom of the grafted molecule are important parameters determining the extent of mechanical interlocking and molecular entanglement in the matrix, eventually determining IFSS. ${ }^{\mathbf{2 0}, 21}$ As the rigidity and molecular length increases, the grafted molecule can better penetrate into the matrix intensifying these effects. In light of these findings, we have also synthesized a range of functionalized carbon fibre derivatives according to Fig. 4. Although there is a slight increase with increasing molecular length and rigidity (aromatic structure), it does not have an appreciable effect in our case.

Significant increase could be obtained for the 4-(trifluoromethyl)benzyl benzoate-functionalized derivative (Fig. 4). In this case the rigid aromatic ring is substituted with a highly lipophilic and yet compact (less steric hindrance compared to a long alkyl chain) trifluoromethyl group. This special structural design allows the grafted molecule to easily penetrate to the $\mathrm{CP}$ matrix and exerts its effect through physical interlocking. It follows that lipophilicity as well as steric effects are crucial factors in case of the CP matrix.

Optical micrographs were taken to investigate the mechanism of micromechanical failure (ESI Material, Fig. S26-28†). 


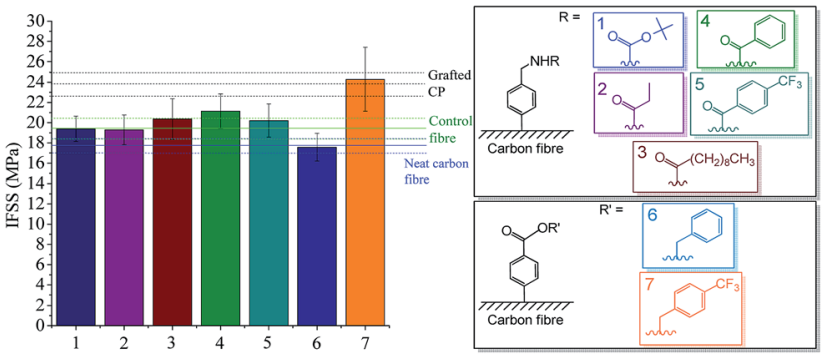

Fig. 4 Interfacial shear strength (IFSS) of carbon fibre reinforced cellulose composites - effect of side chain structure.

The images show that delamination is the dominating failure mode, matrix cracks are expected to appear at higher interfacial shear strength. ${ }^{29}$

Furthermore, to have a deeper insight into the failure mechanism, SEM images of the fracture surfaces of the single fibre composites after the fragmentation test were recorded (only samples elongated until the breakage were analysed), the results are shown in the ESI Material (pp. S32-38†). The SEM images also indicate that delamination is the dominating type of micromechanical failure. While for the cellulose derivativecoated carbon fibre the hole surrounding the fibre is the smallest and some polymer on the carbon fibre surface can also be noticed, for the benzoic acid-functionalized fibre the incompatibility with the matrix is apparent.

Electrochemical grafting. In order to aim at industrial realization, a robust electrochemical process was applied. Electrochemical grafting of the diazonium salt was performed in case of some promising derivatives and thereafter, IFSS was determined as an ultimate measure of the success of the electrochemical procedure.

The cyclic voltammogram obtained for electrochemical grafting of the diazonium salt of $4-[(N$-Boc $)$ aminomethyl $]$ aniline is shown in Fig. S29. $\dagger$ The first electrochemical cycle exhibits a broad irreversible reduction peak typical for diazonium species of this type, having a peak with similar characteristics between $-0.4 \mathrm{~V}$ to $-0.8 \mathrm{~V} \cdot{ }^{19}$ In the second cycle still some reduction occurs, however, to a much smaller extent. The next passes indicate that practically saturation of the surface takes place. We decided not to fully saturate the carbon fibre surface as it is expected to have a diminishing effect on surface roughness and therefore, we only applied one cycle when preparing the functionalized sample for IFSS experiments.

After the electrochemical grafting, the functionalized fibres were further derivatized according to the synthetic procedures shown in the ESI Material, to afford the samples shown in Fig. S30. $\dagger$

The electrochemical grafting experiment results in a 4(aminomethyl)benzene-functionalized carbon fibre composite with similar IFSS compared to the sample produced through purely chemical procedure (see Fig. 3 and S30†). In case of the cellulose propionate-functionalized carbon fibre composite the IFSS decreases appreciably. The underlying reason might be a higher surface coverage of initially grafted molecules that might eventually lead to an "overcrowded" CP coating with less roughness and limited amount of secondary interactions. From this point of view, the electrochemical process needs to be carefully optimized on a large scale application.

\section{Conclusions}

Currently applied carbon fibre reinforced polymers cannot be considered as green materials based on their entirely manmade matrix. By placing the interfacial behaviour of a carbon fibre reinforced cellulose propionate composite under scrutiny, the authors intend to take a step forward to introduce bio-based CFRPs as future alternatives.

In order to design a molecular structure that is able to penetrate into the cellulose propionate matrix efficiently and exert its effect via molecular entanglement and mechanical interlocking, steric factors, lipophilicity of the grafted structure and possible secondary interactions need to be taken into account. Enhanced interfacial shear strength was achieved in case of the cellulose propionate-functionalized carbon fibre composite. Furthermore, similarly high IFSS was also obtained for a grafted molecule possessing highly lipophilic and yet compact $-\mathrm{CF}_{3}$ substituent, and in case of a molecule when hydrogen bonding with the CP matrix could possibly take place.

It should also be pointed out that this study is the first providing synthetic strategy for covalently binding a cellulose derivative to a largely graphitic surface, thereby opening the gates to functionalize many other carbonaceous materials for innovative applications.

\section{Conflicts of interest}

There are no conflicts to declare.

\section{Acknowledgements}

The authors thank the COI program "Construction of nextgeneration infrastructure using innovative materials - Realization of a safe and secure society that can coexist with the Earth for centuries" supported by MEXT and JST. This study was also supported in part by the Advanced Low Carbon Technology Research and Development Program (ALCA) and the Crossministerial Strategic Innovation Promotion Program (SIP) of the JST.

\section{Notes and references}

1 S. Das, J. Warren, D. West and S. M. Schexnayder, Global Carbon Fiber Composites Supply Chain Competitiveness Analysis, Technical Report ORNL/SR-2016/100-NREL/TP-6A5066071, 2016.

2 J. Schultz, L. Lavielle and C. Martin, J. Adhes., 1987, 23, 45.

3 M. R. Piggott, Carbon, 1989, 27, 657.

4 J. D. H. Hughes, Compos. Sci. Technol., 1991, 41, 13.

5 L. T. Drzal and M. Madhukar, J. Mater. Sci., 1993, 28, 569.

6 M. Sharma, S. Gao, E. Mäder, H. Sharma, L. Y. Wei and J. Bijwe, Compos. Sci. Technol., 2014, 102, 35. 
7 J. Karger-Kocsis, H. Mahmood and A. Pegoretti, Prog. Mater. Sci., 2015, 73, 1.

8 L. Ma, L. Meng, D. Fan, J. He, J. Yu, M. Qi, Z. Chen and Y. Huang, Appl. Surf. Sci., 2014, 296, 61.

9 L. Ma, L. Meng, Y. Wang, G. Wu, D. Fan, J. Yu, M. Qi and Y. Huang, RSC Adv., 2014, 4, 39156.

10 Y. Wang, L. Meng, L. Fan, L. Ma, M. Qi, J. Yu and Y. Huang, Appl. Surf. Sci., 2014, 316, 366.

11 Y. Wang, L. Meng, L. Fan, G. Wu, L. Ma, M. Zhao and Y. Huang, Appl. Surf. Sci., 2016, 362, 341.

12 K. Shiba, M. Tagaya, S. Samitsu and S. Motozuka, ChemPlusChem, 2014, 79, 197.

13 C. U. Pittman, Z. Wu, W. Jiang, G. R. He, B. Wu, W. Li and S. D. Gardner, Carbon, 1997, 7, 929.

14 D. A. Buttry, J. C. M. Peng, J. B. Donnet and S. Rebouillat, Carbon, 1999, 37, 1929.

15 G. J. Ehlert, Y. Lin and H. A. Sodano, Carbon, 2011, 49, 4246. 16 M. Delamar, G. Désarmot, O. Fagebaume, R. Hitmi, J. Pinson and J. M. Savéant, Carbon, 1997, 35, 801.

17 K. M. Beggs, L. Servinis, T. R. Gengenbach, M. G. Huson, B. L. Fox and L. C. Henderson, Compos. Sci. Technol., 2015, 118, 31.

18 L. Servinis, L. C. Henderson, L. M. Andrighetto, M. G. Huson, T. R. Gengenbach and B. L. Fox, J. Mater. Chem. A, 2015, 3, 3360.

19 L. Servinis, K. M. Beggs, C. Scheffler, E. Wölfel, J. D. Randall, T. R. Gegenbach, B. Demir, T. R. Walsh, E. H. Doeven, P. S. Francis and L. C. Henderson, Carbon, 2017, 118, 393.

20 L. Servinis, K. M. Beggs, T. R. Gengenbach, E. H. Doeven, P. S. Francis, B. L. Fox, J. M. Pringle, C. Pozo-Gonzalo, T. R. Walsh and L. C. Henderson, J. Mater. Chem. A, 2017, 5, 11204.

21 B. Demir, L. C. Henderson and T. R. Walsh, ACS Appl. Mater. Interfaces, 2017, 9, 11846.

22 J. He, Y. Huang, L. Meng, H. Cao and H. Gu, J. Appl. Polym. Sci., 2009, 112, 3380.
23 J. M. Henshaw, W. Han and A. D. Owens, J. Thermoplast. Compos. Mater., 1996, 9, 4.

24 X. Zhang, H. Xu and X. Fan, RSC Adv., 2014, 4, 12198.

25 D. Liu, Y. Zhu, J. Ding, X. Lin and X. Fan, Composites, Part B, 2015, 77, 363.

26 D. Klemm, B. Heublein, H. P. Fink and A. Bohn, Angew. Chem., Int. Ed., 2005, 44, 3358.

27 K. J. Edgar, C. M. Buchanan, J. S. Debenham, P. A. Rundquist, B. D. Seiler, M. C. Shelton and D. Tindall, Prog. Polym. Sci., 2001, 26, 1605.

28 S. Banerjee, T. Hemraj-Benny and S. S. Wong, Adv. Mater., 2005, 17, 17.

29 L. Servinis, PhD thesis, Deakin University, 2014.

30 N. Li, Z. Wu, L. Huo, L. Zong, Y. Guo, J. Wang and W. Jian, RSC Adv., 2016, 6, 70704.

31 R. Kakuchi, R. Ito, S. Nomura, H. Abroshan, K. Ninomiya, T. Ikai, K. Maeda, H. J. Kim and K. Takahashi, RSC Adv., 2017, 7, 9423.

32 A. Kelly and W. R. Tyson, J. Mech. Phys. Solids, 1965, 13, 329. 33 T. Ohsawa, A. Nakayama, M. Miwa and A. Hasegawa, J. Appl. Polym. Sci., 1978, 22, 3203.

34 A. P. Kettle, A. J. Beck, L. O'Toole, F. R. Jones and R. D. Short, Compos. Sci. Technol., 1997, 57, 1023.

35 K. Naito, Fracture Behaviour of Continuous Carbon Fibre, in Improvement of Resin Impregnation Property and Reliability Evaluation of CFRP (Carbon Fibre Reinforced Plastic), Technical Information Institute Co, Ltd, Tokyo, 2010, vol. 34, http://www.gijutu.co.jp/weblibraryadv/webb_1581.htm.

36 J. F. King, R. Rathore, J. Y. L. Lam, Z. R. Guo and D. F. Klassen, J. Am. Chem. Soc., 1992, 114, 3028.

37 G. Zhang, S. Sun, D. Yang, J.-P. Dodelet and E. Sacher, Carbon, 2008, 46, 196.

38 T. Jawhari, A. Roid and J. Casado, Carbon, 1995, 33, 1561.

39 J. Tiller, P. Berlin and D. Klemm, Macromol. Chem. Phys., 1999, 200, 1.

40 G. Nansé, E. Papirer, P. Fioux, F. Moguet and A. Tressaud, Carbon, 1997, 35, 175. 